\title{
A STUDY ON EFFECT OF AEROBIC AND PELVIC FLOOR EXERCISES IN PERIMENOPAUSAL HOUSEWIVES
}

\author{
Ruchi Mishra ${ }^{1}$, Sanjeev K Jha ${ }^{2}$, Fatema Fidwi ${ }^{3}$, Deepa Sethiya ${ }^{4}$, Soumitra Sethia *5. \\ ${ }^{1}$ Associate Professor, Ujjain College of Physiotherapy, Ujjain, Madhya Pradesh, India. \\ ${ }^{2}$ Professor, Ujjain College of Physiotherapy, Ujjain, Madhya Pradesh, India. \\ ${ }^{3}$ Physiotherapist, Madhya Pradesh, India. \\ ${ }^{4}$ Assistant Professor, Ujjain College of Physiotherapy, Ujjain, Madhya Pradesh, India. \\ ${ }^{*}$ Assistant Professor, Department of Community Medicine, Government Medical College, Khandwa, \\ Madhya Pradesh, India.
}

\section{ABSTRACT}

Background: Perimenopause, not only a transitional state from reproductive adulthood to menopause but an emotional and physical U-turn of abrupt changes. Research studies have ascertained risk of depression and irritability due to fluctuating hormone levels which causes severe stress in women. This study emphasizes on the community of females who are more prone to such stress and ignorance due to their social inactiveness, so the study was conducted amongst thecommunity females of perimenopausal state to check the effectiveness of aerobic and strengthening training.

Aim: The present study aims to assess the effect of aerobic exercises and pelvic floor exercises in perimenopausal housewives.

Methods: In present experimental study, total of 30 females of age b/w 40-50 years with perimenopausal symptoms were randomly selected from the department of physiotherapy in R.D.G.M.C. Ujjain for up to 2 months of study. Pre-readings of menopause rating scale were taken and compared to the post readings after 2 months treatment sessions with various aerobic and pelvic floor exercises. Data analysis was performed using paired ttest with significance level of the test waspd" 0.05 the results showed that both postnatal depression and insomnia decreases significantly after two weeks $(p=0.001)$.

Conclusion: This study revealed that physical activity help perimenopausal women to increase their quality of life and to cope up with complaints related to perimenopausal symptoms.

KEY WORDS: Perimenopausal, Pelvic floor exercises, physiotherapy,

Address for correspondence: Dr.SoumitraSethia, Assistant Professor, Department of Community Medicine, Government Medical College, Khandwa, Madhya Pradesh, India.

E-Mail: drsoumitrasethia@gmail.com

Access this Article online Quick Response code

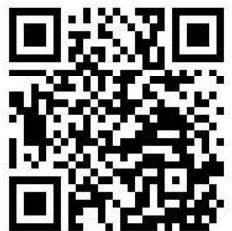

DOI: $10.16965 /$ ijpr.2019.200



Received: 15 Nov 2019

Peer Review: 15 Nov 2019

Revised: None
Journal Information

International Journal of Physiotherapy and Research

ICV for 2016 ISSN (E) 2321-1822 | ISSN (P) 2321-8975

https://www.ijmhr.org/ijpr.html

DOI-Prefix: https://dx.doi.org/10.16965/ijpr

Article Information

Accepted: 12 Dec 2019

Published (O): 11 Feb 2020

Published (P): 11 Feb 2020

\section{INTRODUCTION}

Perimenopause is a period of transition from reproductive adulthood to menopause [1].Many women experience an array of symptoms as their hormones shift during the months or years leading up to menopause - that is, the natural end of menstruation. Menopause is a point in time, but perimenopause (peri, Greek for "around" or "near" + menopause) is an extended transitional state. It's also sometimes referred to as the menopausal transition, although technically, the transition ends 12 months earlier than 
perimenopause.

Perimenopause has been variously defined, but experts generally agree that it begins with irregular menstrual cycles - courtesy of declining ovarian function - and ends a year after the last menstrual period. Perimenopause varies greatly from one woman to the next. The average duration is three to four years, although it can last just a few months or extend as long as a decade. Some women feel buffeted by hot flashes and wiped out by heavy periods; many have no bothersome symptoms. Periods may end abruptly for some, while others may menstruate erratically for years. The physical changes of perimenopause are rooted in hormonal alterations, particularly variations in the level of circulating estrogen. During our peak reproductive years, the amount of estrogen in circulation rises and falls predictably throughout the menstrual cycle. Estrogen levels are largely controlled by two hormones, folliclestimulating hormone (FSH) and luteinizing hormone (LH) [2].

Most of women in perimenopause suffer from urinary incontinence. Physical therapy interventions such as pelvic floor exercises are one of the most effective and low risk solutions for urinary incontinence. It helps to retrain the bladder and strengthen the pelvic floor muscles to help to resolve problem [1].Women in perimenopause also have symptoms like breast tenderness, fatigue, irregular periods, urinary leakage when coughing or sneezing, urinary urgency, mood swings, trouble sleeping [3-6]. Epidemiological study indicate that the risks of depression are greatest in the reproductive years, when harmonal levels fluctuates during the menstrual cycles and the symptoms irritability,nervousness and dysphoria increased in menopause transition is also due to fluctuations or rates of change in estradiol showing depressive symptoms in vulnerable women[3]. Vasomotor symptoms affect up to $75 \%$ of perimenopausal women $[4,5]$.

Hot flashes not only disturb women at work and interrupt daily activities but also disrupt sleep.Insomnia occurs in 40-50\% of women in menopause transition.Women with insomnia are likely to report problem such as anxiety, stress, tension and depressive symptoms.
Working women may require preferably more care due to dual role responsibility. It may become slightly difficult to manage all activities with same efficient way as before.That may cause feeling of guilt, limitation, stress etc. In these working women physical relaxation, emotional support and essential care needed for healthy living [4].

Between 45 and 55 years of age,women experience changes in roles, responsibilities and relationships which accompany ageing in general., Children leave home for short periods of time.These episodes cause stress.The effect of this change most find it difficult to cope.

Housewives are socially inactive they are not allowed to go places.They do not have friend circles they are only limited to their homes also they do not have awareness of menopause and perimenopausal symptoms so in Housewives community the ladies suffer more from such symptoms and didn'tknow how to deal with them [7-9].The study was aimed to assess the effect of aerobic and pelvic floor exercise in perimenopausal women. The objectives of the study were 1) To assess the decrease in postmenopausal symptoms, 2) To assess the improvement in psychological health, 3)To assess the increase in pelvic muscle strength, 4) To assess the improvement in quality of life of the study participants.

\section{METHODOLOGY}

An intervention study was planned to achieve the aims and objective. The opportunistic sampling method was used to register the study participants. Study area or study participants were Patients from department of physiotherapy in R.D.G.M.C. Ujjain and home visit. A total of 30 participants were followed for a period of 2 months. The study participants were registered with inclusion criteria as A) age between 40-50 years, B) perimenopausal symptoms 1) hot flashes, 2) night sweats, 3)insomnia, 4) irritability. Patient selected based onMRS.(irregular menstrual cycle,urinary incontinence) the exclusion criteria were women treated with HRT and non-hormonal climacteric medicine within past 2 weeks before the study, hysterectomy, oophorectomy, having regular menstruation. The outcome was measured by menopause rating 
scale (MRS). In this study firstly the questionnaire of menopause rating scale (MRS) was distributed to females fulfilling the inclusion and exclusion criteria,then patients showing perimenopausal symptoms scoring less than 22 on the basis of MRS scale and having severe grade were included. 30 females were selected based on this parameter of age between 41-50yrs,exercise protocol and the purpose of this treatment was explained to the patient. Consent form was taken from the patient. The total duration of treatment is $\mathbf{5 0}$ mins per day and 6 sitting per week. Total duration of study is 2 months. First assessment was taken at the starting of the treatment and 2 nd after the treatment which showed significant improvement in functional capacity,mood swing and irritability. Exercise protocol to alleviate the symptoms of perimenopause: (total duration:50mins)

\section{For relaxation-}

1. a. Breathing exercise -(5-6 reps)(3mins)

- Diaphramatic breathing exercise

- Pursed lip breathing

b. Yoga (5 reps. $10 \mathrm{sec}$ hold) (10mins)

- Uttasana [forward bend]

- Navasana [boat pose]

- Dhanurasana [bow pose]

- Anuvittasana [standing back bend]

(relax time:1min)

2. To improve core strength(10 reps. $10 \mathrm{sec}$ hold) (5 mins)

- Back extension exercise

- Leg lifts

- Cat and camel exercise

(relax time: $1 \mathrm{~min}$ )
3. To improve pelvic strength:-(10 reps $10 \mathrm{sec}$ hold)(10mins)

- Keigles

- Squats

- Bridges (relax time:1min)

4. To improve or maintain posture, balance, spinal alignment and flexibility:-( 5 reps.) (5mins)

- Wall slide

- Leg raise

- Stretching- hams, quads and calf

(relax time:2mins)

5. For cardiac fitness:-

- Spot jogging(3 mins)

- Stair climbing(3 mins)

- Walking(5mins)

6. Cool down exercise:-(1min)

breathing exercise

\section{RESULTS}

The study had a total of 30 participants and the study was carried out over a duration of 2 months. The initial MRS score was calculated, and MRS scoring was performed after 2 months of physiotherapeutic intervention. The mean age of study participants was 44.96 years $( \pm 2.1)$ and mean MRS score at the start of study was 36.3 $( \pm 5.2)$. Mean MRS score after 2 months of intervention came out to be14.1 (7.7) and the mean improvement was $23.3( \pm 3)$.

The above table depicts the MRS score for each question's before and after the physiotherapeutic intervention. The improvement for each question is highly significant indicating that the intervention plays a significant role in improving patients perimenopausal symptoms and aid her to cope up with the state.

\begin{tabular}{|c|c|c|c|c|c|}
\hline Question & $\begin{array}{c}\text { MRS before } \\
\text { intervention } \\
\text { (Mean) }\end{array}$ & $\begin{array}{c}\text { MRS after } \\
\text { Intervention } \\
\text { (Mean) }\end{array}$ & Improvement & $\begin{array}{c}\text { Confidence } \\
\text { Interval }\end{array}$ & $\mathbf{p}$ Value \\
\hline $\mathbf{1}$ & 3.2 & 1.03 & 2.2 & $1.9-2.4$ & 0 \\
\hline $\mathbf{2}$ & 3.6 & 1.2 & 2.4 & $2.0-2.6$ & 0 \\
\hline $\mathbf{3}$ & 3.2 & 1.2 & 2 & $1.6-2.2$ & 0 \\
\hline $\mathbf{4}$ & 3.3 & 1.6 & 1.7 & $1.3-2.0$ & 0 \\
\hline $\mathbf{5}$ & 3.3 & 1.2 & 2.1 & $1.7-2.4$ & 0 \\
\hline $\mathbf{6}$ & 3.6 & 1.2 & 2.4 & $2.1-2.6$ & 0 \\
\hline $\mathbf{7}$ & 3.4 & 1.2 & 2.2 & $1.9-2.5$ & 0 \\
\hline $\mathbf{8}$ & 3.3 & 1.5 & 1.8 & $1.5-2.1$ & 0 \\
\hline $\mathbf{9}$ & 3.6 & 1.1 & 2.5 & $2.1-2.8$ & 0 \\
\hline $\mathbf{1 0}$ & 3.3 & 1.1 & 2.2 & $1.7-2.5$ & 0 \\
\hline
\end{tabular}




\section{DISCUSSION}

Perimenopause or menopause transition begins several years before menopause. It's a time when ovaries gradually began to make less estrogen it usually starts in a women with 40.The average length of perimenopause is 4 years but for some women this stage may last only a few months or continue for 10 years. Women in perimenopause experience symptoms like hot flashes, breast tenderness, irregular periods, mood swings and trouble sleeping [8].

In this study it was found that exercise training can reduce the perceived intensity of menopausal symptoms and increase the state of being psychologically fine. The fact that dealing with women in menopausal period gets women adopt different habits and lifestyle in this critical period will be helpful in overcoming symptoms related to menopausal period.

In this study 30 females were included in this study on the basis MRS scale,exercise training program included in this study were relaxation exercises like breathing ex. and yoga poses, core stability and pelvic muscle strengthening exercise to increase pelvic muscle strength and urinary incontinence and jogging, running and walking to improve cardiac fitness and make psychologically fit.In the training programme assessment taking after exercise session proved that exercise training might increase the cardiovascular suitability of perimenopausal women.It is stated in various studies and database that physical activity and the participation in exercise have some significant positive effect on symptoms related to menopause [7].

At the end of a study the effect of exercise training on menopausal symptoms was analysed in general terms based on changes in MRS scale.This shows that exercise have a positive impact on menopausal symptoms, psychological health and quality of life [7].

\section{CONCLUSION}

This study revealed that physical activity help women to increase their quality of life and to cope up with complaints related to perimenopausal symptoms.

\section{Conflicts of interest: None}

\section{REFERENCES}

[1]. Effects of pelvic floor muscle training vs an assisted pelvic floor muscle training among rural perimenopausal women with urinary incontinence: A comparative study ReemaVyas, Dr.DeepaliHande ,PrandnyaNishad, HeerBarnot(1Jan2018) International Journal of Yoga, Physiotherapy and Physical Education. 2018;3(1):121-124.

[2]. Alth.harvard.edu/newsletters/Harvard_ Womens_ Health_Watch.h(June2009)

[3]. Ellen.W.FreemanPhd,Clarisa.R.Gracia.M.D.(2004)

[4]. SlultanAlanPhD, RN, EbruGozuyesil, PhD, RN, SuleGokyildiz, PhD, RN. Effects of Menopause on the Life of Women Who Experience Hot Flashes and their Health-Seeking Behaviours. International Journal of Caring Sciences 2016;9(2):542.

[5]. Postmenopausal syndrome. Pronob K. Dalal and Manu Agarwal Indian J Psychiatry. 2015 Jul; 57(Suppl 2): S222-S232.

[6]. The Impact of Exercise on Perimenopause by Maria Luque on Mar 27, 2013, https://www.ideafit.com/ fitness-library/the-impact-of-exercise-onperimenopause.

[7]. Ays 'egulA gýl, Faruk Abýke, Arzu Das 'kapan, RýdvanAlaca, and Handan Tuz un . Short-Term Exercise Approaches on Menopausal Symptoms, Psychological Health, and Quality of Life in Postmenopausal Women. Hindawi Publishing Corporation Obstetrics and Gynecology International. 2010, Article ID 274261, 7 pages doi:10.1155/2010/27426.

[8]. https://www.webmd.com/menopause/guide/guideperimenopause.

[9]. Perimenopausal risk factors and future health The ESHRE Capri Workshop Group Human Reproduction Update, 2011;17(5):706-717. https://doi.org/ 10.1093/humupd/dmr020

[10]. Capri, T. E.; Cuzick, J.; Glasier, A.; La Vecchia, C.; Maragore, D. M.; Negri, E.; Rossi, M.; Spector, T.; Trichopoulos, D.; VAN Baak, m. a.; Zocchetti, C.; Baird, D. T.; Banagiano, G.; Crosignani, P. G.; Diczfalusy, E.; Evers, J. L. H.; Gianaroli, L.; Volpe, Annibale. Perimenopausal risk factors and future health $-\mathrm{In}$ : HUMAN REPRODUCTION UPDATE. - ISSN 1355-4786. - STAMPA.2011;17:706-717.

How to cite this article:

Ruchi Mishra, Sanjeev K Jha, Fatema Fidwi, Deepa Sethiya, Soumitra Sethia. A STUDY ON EFFECT OF AEROBIC AND PELVIC FLOOR EXERCISES IN PERIMENOPAUSAL HOUSEWIVES. Int J Physiother Res 2020;8(1):3350-3353. DOI: 10.16965/ijpr.2019.200 